\title{
Effect of antibiotic pre-treatment and pathogen challenge on the intestinal microbiota in mice
}

\author{
Tadasu lizumi ${ }^{1,5^{*}} \mathbb{D}$, Takako Taniguchi ${ }^{2}$, Wataru Yamazaki ${ }^{2}$, Geraldine Vilmen ${ }^{3}$, Alexander V. Alekseyenko ${ }^{1}$,
} Zhan Gao', Guillermo I. Perez Perez ${ }^{1}$ and Martin J. Blaser ${ }^{1,4}$

\begin{abstract}
Background: More than 50 years after the discovery of antibiotics, bacterial infections have decreased substantially; however, antibiotics also may have negative effects such as increasing susceptibility to pathogens. An intact microbiome is an important line of defense against pathogens. We sought to determine the effect of orally administered antibiotics both on susceptibility to pathogens and on impact to the microbiome. We studied Campylobacter jejuni, one of the most common causes of human diarrhea, and Acinetobacter baumannii, which causes wound infections. We examined the effects of antibiotic treatment on the susceptibility of mice to those pathogens as well as their influence on the mouse gut microbiome.
\end{abstract}

Results: In C57/BL6 mice models, we explored the effects of pathogen challenge, and antibiotic treatment on the intestinal microbiota. Mice were treated with either ciprofloxacin, penicillin, or water (control) for a 5-day period followed by a 5-day washout period prior to oral challenge with C. jejuni or A. baumannii to assess antibiotic effects on colonization susceptibility. Mice were successfully colonized with C. jejuni more than 118 days, but only transiently with A. baumannii. These challenges did not lead to any major effects on the composition of the gut microbiota. Although antibiotic pre-treatment did not modify pathogen colonization, it affected richness and community structure of the gut microbiome. However, the antibiotic dysbiosis was significantly reduced by pathogen challenge.

Conclusions: We conclude that despite gut microbiota disturbance, susceptibility to gut colonization by these pathogens was unchanged. The major gut microbiome disturbance produced by antibiotic treatment may be reduced by colonization with specific microbial taxa.

Keywords: Microbiome, Antibiotic treatment, Pathogen challenge, Penicillin, Ciprofloxacin, Campylobacter jejuni, Acinetobacter baumannii, High-throughput sequencing, Next-generation sequencing

\section{Background}

The mammalian intestine hosts a complex and diverse microbial community $[1,2]$. This ecosystem interacts extensively with its host, with substantial physiological and pathological effects [3]. For example, the gut microbiota is crucial to the host's ability to resist colonization by pathogens $[4,5]$, although the mechanisms involved are incompletely characterized [6].

*Correspondence: Tadasu.lizumi@nyumc.org

5026 W VAMC, 423 East 23th Street, New York, NY 10010, USA

Full list of author information is available at the end of the article
The clinical use of antibiotics has become massive in recent decades [7]. Their use increases susceptibility to acquired pathogen, although the underlying mechanisms are not well-understood [8]. Antibiotics change the composition of microbiota in the GI tract [9], affecting metabolic, hormonal, and immunological interactions between community and host, as well as intra-community interactions [10-12]. Separately, or together, these effects may increase host susceptibility to infection by introduced pathogens.

Campylobacter jejuni are Gram-negative, microaerophilic, curved rods, that commonly cause diarrheal 
illnesses, and can affect previously healthy hosts [13-15]. Acinetobacter baumannii, non-fermentative Gramnegative cocobacilli, have become increasingly common nosocomial pathogens, especially in intensive care units (ICUs) $[16,17]$. The high prevalence of intestinal A. baumannii colonization in ICU patients suggests that the colon may be an important reservoir [18].

In this study, we developed mouse models involving colonization with these human pathogens to address three questions germane to colonization resistance; (1) what is the extent to which pathogens such as $C$. jejuni or A. baumannii colonize the GI tract of mice; (2) how does such colonization affect the gut microbiota; and (3) does pre-treatment with antibiotics change microbiota compositions and affect susceptibility to colonization by these pathogens?

\section{Methods}

\section{Study design}

Three related experiments were conducted in which control (untreated) mice were compared to experimental mice that were challenged by a pathogen, either alone, or in conjunction with antibiotic pre-treatment. In experiment \#1, 7 week-old mice were challenged with either $A$. baumannii or C. jejuni (Additional file 1: Figure S1, panel A). In experiment \#2, 6 week-old mice were challenged with one of three strains of $C$. jejuni that varied based on their mouse-passage histories (Additional file 1: Figure S1, panel B). In experiment \#3, mice were exposed first to an antibiotic regime of either penicillin or ciprofloxacin or neither (control), and then were challenged with either $A$. baumannii or C. jejuni, or remained unchallenged (Additional file 1: Figure S2).

\section{Mice}

Female C57BL/6NJ mice were obtained from Jackson Laboratories at $\sim 5$ to 6 weeks of age and allowed to adjust to the NYU animal facility for 1 week. The animals then were used in experiment \#1 (Additional file 1: Figure S1, panel A). In addition, animals originally received from Jackson Laboratories were used for breeding at the NYU animal facility, and the offspring females were used for experiments \#2 and 3 (Additional file 1: Figure S1, panel B). In experiment \#3, 10 days prior to bacterial challenge, mice were given water containing penicillin VK $(0.167 \mathrm{mg} / \mathrm{ml}$; Sigma Aldrich, St Louis MO, USA), or ciprofloxacin $(0.13 \mathrm{mg} / \mathrm{ml}$; Acros Organics, Geel, Belgium), or no antibiotic (control) for 5 days. Water containers were changed twice during these 5 days to supply fresh antibiotics. The protocols for the mouse experiments included in this study were approved by the New York University School of Medicine Institutional Animal Care and Use Committee (IACUC).

\section{Bacterial strains used for mice inoculation}

Campylobacter jejuni strain 81-176, that was originally isolated from a milkborne outbreak of human campylobacteriosis [19], and has been used in human volunteer studies [20], was used in all three experiments. For experiment $\# 2$, we used two additional $C$. jejuni strains that were recovered from mice experimentally inoculated with strain 81-176 in experiment \#1. Campylobacter jejuni strain MP-10 was isolated from mouse stool 42 days after colonization, and C. jejuni strain Cecum J1 was isolated from the cecum of a mouse 119 days after colonization. All C. jejuni strains were cultured for $48 \mathrm{~h}$ on Skirrow agar (Becton-Dickinson, Franklin Lakes NJ, USA) under microaerobic conditions at $37{ }^{\circ} \mathrm{C}$. Cultures then were resuspended in phosphate-buffered saline (PBS; pH 7.2) and adjusted to a concentration of $10^{8} \mathrm{CFU}$ (by $\mathrm{OD}_{600}$ ) in $400 \mu \mathrm{l}$, which was introduced via oral gavage to test mice. Control mice received an oral gavage of $400 \mu \mathrm{l}$ of PBS.

Acinetobacter baumannii strain 11-1, used in experiments \#1 and 3, was a recent clinical isolate obtained from the New York University Langone Medical Center (NYULMC) Clinical Microbiology Laboratory. Acinetobacter baumannii was cultured for $24 \mathrm{~h}$ using Columbia sheep blood agar (BD) and CHROM agar Acinetobacter Base (DRG International, Springfield NJ, USA) under aerobic conditions at $37{ }^{\circ} \mathrm{C}$. Acinetobacter baumannii was resuspended in PBS and adjusted to a concentration of $1.3 \times 10^{11} \mathrm{CFU}$ (by $\mathrm{OD}_{600}$ ) per $400 \mu$ l to create the oral gavage inoculum.

\section{Fecal specimen collection, culture and DNA extraction}

In all experiments, fecal specimens were collected from mice before and after pathogen challenge (Additional file 1: Figures S1, S2), and were either immediately cultured or frozen at $-20{ }^{\circ} \mathrm{C}$. About $20 \mathrm{mg}$ of feces were resuspended in $1 \mathrm{ml}$ of PBS and vortex-mixed for $30 \mathrm{~s}$ at room temperature. From this stock suspension, tenfold dilutions were made in PBS. Aliquots $(100 \mu \mathrm{l})$ of the $10^{\circ}$, $10^{-2}$, and $10^{-3}$ dilutions were plated on CHROM agar for $A$. baumannii and on Skirrow agar for C. jejuni. Plates were incubated under the conditions indicated above and colony counts were reported as CFU/mg stool. Fecal DNA also was extracted from a $20 \mathrm{mg}$ aliquot of mouse feces using the PowerSoil DNA Isolation Kit (MOBIO, West Carlsbad CA, USA), according to the manufacturer's protocol. The concentration of extracted DNA was determined by Nanodrop 1000 (Thermo Scientific, Watham MA, USA), and DNA was stored at $-20^{\circ} \mathrm{C}$ until used.

\section{Quantitative PCR}

Sets of qPCR primers (Table 1) were used to quantitate bacterial populations, based on the universal bacterial 
Table 1 Primers used for PCR in this study

\begin{tabular}{lll}
\hline Target & Primer designation & Primer sequence $^{\mathbf{a}}$ \\
\hline Total bacteria & $519 \mathrm{~F}$ & GGACTACCVGGTATCTAAKCC \\
16S rRNA & $785 \mathrm{R}$ & CAGCAGCCGCGGTRATA \\
C. jejuni & $\mathrm{F}$ & AGCGATCAAAGCAAAATTCC \\
luxS & $\mathrm{R}$ & GGCAATTTGTTTGGCTTCAT \\
A. baumannii & $\mathrm{F}$ & TTTAGCTCGTCGTATTGGACTTGA \\
Oxa-51 & $\mathrm{R}$ & GCCTCTTGCTGAGGAGTAATTTTT \\
\hline $\mathrm{a} \mathrm{V}=\mathrm{A}+\mathrm{C}+\mathrm{G}, \mathrm{K}=\mathrm{G}+\mathrm{T}, \mathrm{R}=\mathrm{A}+\mathrm{G}$ &
\end{tabular}

$16 \mathrm{~S}$ rRNA sequences [21], C. jejuni luxS [22], and A. baumannii oxa51 [23]. qPCRs were performed using $3.5 \mathrm{mM}$ $\mathrm{MgCl}_{2}, 0.4 \mathrm{ng} / \mu \mathrm{l}$ bovine serum albumin, $0.2 \mathrm{mM}$ of each deoxynucleoside triphosphate, $10 \mathrm{pmol}$ of each primer, 0.625 U Taq DNA polymerase (Qiagen, Valencia CA, USA), and $2 \mu \mathrm{l}$ extracted DNA in a final $20-\mu$ l volume of SYBR green master mix. qPCR conditions included 5 min at $94{ }^{\circ} \mathrm{C}$ and 45 cycles of $10 \mathrm{~s}$ at $94{ }^{\circ} \mathrm{C}, 10 \mathrm{~s}$ at $60^{\circ} \mathrm{C}$ (C. jejuni and A. baumannii) or $56^{\circ} \mathrm{C}$ (total bacteria). All assays were performed using a Light Cycler 480 (Roche Diagnostic Corporation, Indianapolis IN, USA). Bacterial numbers were determined using standard curves based on serial dilutions of cloned PCR products. Each sample was tested at least twice, and the results were analyzed using the Rotor-Gene 3000 v.6.1.81 software.

Library preparation for high-throughput sequencing (HTS) All samples were amplified and barcoded for multiplex pyrosequencing using primers targeted to the V4 region of the bacterial $16 \mathrm{~S}$ rRNA gene under uniform PCR conditions that included $3 \mathrm{~min}$ at $94{ }^{\circ} \mathrm{C}$ and 45 cycles of $45 \mathrm{~s}$ at $94{ }^{\circ} \mathrm{C}, 60 \mathrm{~s}$ at $50{ }^{\circ} \mathrm{C}$, and $90 \mathrm{~s}$ at $72{ }^{\circ} \mathrm{C}$ with final extension for $10 \mathrm{~min}$ at $72{ }^{\circ} \mathrm{C}$ [24]. We used forward primer (AAT GAT ACG GCG ACC ACC GAG ATC TAC ACT ATG GTA ATT GTG TGC CAG CMG CCG CGG TAA) that includes a $5^{\prime}$ Illumina adaptor, forward primer pad, 2 bp linker and the 515F 16S rRNA primer, and reverse primer (CAA GCA GAA GAC GGC ATA CGA GAT NNNNNNNNNNNN-AGT CAG TCA G-CC-GGA CTA CHV GGG TWT CTA AT) that includes the Illumina $3^{\prime}$ adapter with 12-nt error-correcting Golay barcode, reverse primer pad, 2 bp linker and the 806R $16 \mathrm{~S}$ rRNA primer. We ran PCR in triplicate using $0.2 \mu \mathrm{M}$ of the primers, $1 \mu \mathrm{l}$ of template and $1 \mathrm{X}$ HotMasterMix (5 PRIME, Gaithersburg MD, USA), and cleaned the products using a PCR Purification Kit (Qiagen) after pooling. Cleaned PCR products were quantified using the Qubit dsDNA HS Assay Kit (Invitrogen ${ }^{\mathrm{TM}}$, Eugene OR, USA), then adjusted to an optimal molarity as described. Sequencing was performed using the Illumina MiSeq platform in the NYULMC Genome Technology Core.

\section{Taxonomic and ecological analyses}

We analyzed all sequence data using the QIIME software (version Mac Qiime 1.8.0) [25]. After filtering procedures, similar sequences were clustered into operational taxonomic units (OTUs) using an open reference approach with UCLUST [26] against the Greengenes Core set. A representative sequence was then aligned using PyNAST, and FastTree created phylogenetic trees. Rarefaction analysis used Chao-1 and whole PD to measure $\alpha$-diversity. Unweighted UniFrac distances were calculated to assess $\beta$-diversity; the Unweighted paired group method with arithmetic mean (UPGMA) was performed for UniFrac-based jackknifed hierarchical clustering. Principal coordinates analysis (PCoA) of UniFrac distance matrices provided graphical representation using a KiNG, ANOVA was used to compare OTU and genus-level abundances, and Linear discriminant analysis (LDA) effect size (LEfSe), a tool that can compare differences of relative abundance between $\geq 2$ biological conditions [27], also was used for analysis.

\section{Results \\ Quantifying bacteria in fecal DNA}

Assessing total DNA concentrations using Nanodrop and total bacterial $\log _{10}$ copy number/ng DNA by qPCR, we found that they were similar between the control animals and those treated with either after antibiotic or pathogen challenge (Additional file 1: Figure S3, panels $a-j$ ). Thus, neither the antibiotic treatments nor the pathogen challenge affected the overall population size of the intestinal microbiota.

\section{Assessment of mouse intestinal colonization after challenge}

Although we were able to colonize mice with A. baumannii (Fig. 1, left column), as evaluated by both culture and qPCR, colonization was transient and at low density. In contrast, we could achieve persistent mouse colonization with $C$. jejuni strain 81-176 (for $\geq 15$ weeks), as confirmed by both culture and qPCR, until the experiment ended (Fig. 1, middle and right columns). Thus, the $A$. baumannii and $C$. jejuni strains used differed greatly in their ability to colonize the murine gut.

\section{Assessment of gut microbiome changes associated with C. jejuni challenge and cage effects}

We then assessed the gut microbiota in mice followed prospectively from 6.5 to 23.9 weeks (Fig. 2). In the initial pre-challenge samples, the bacterial communities were nearly identical in their community structure (column A). However, over time, the communities differentiated, based on the cage in which they were housed, and independently based on C. jejuni challenge or not. Next, 


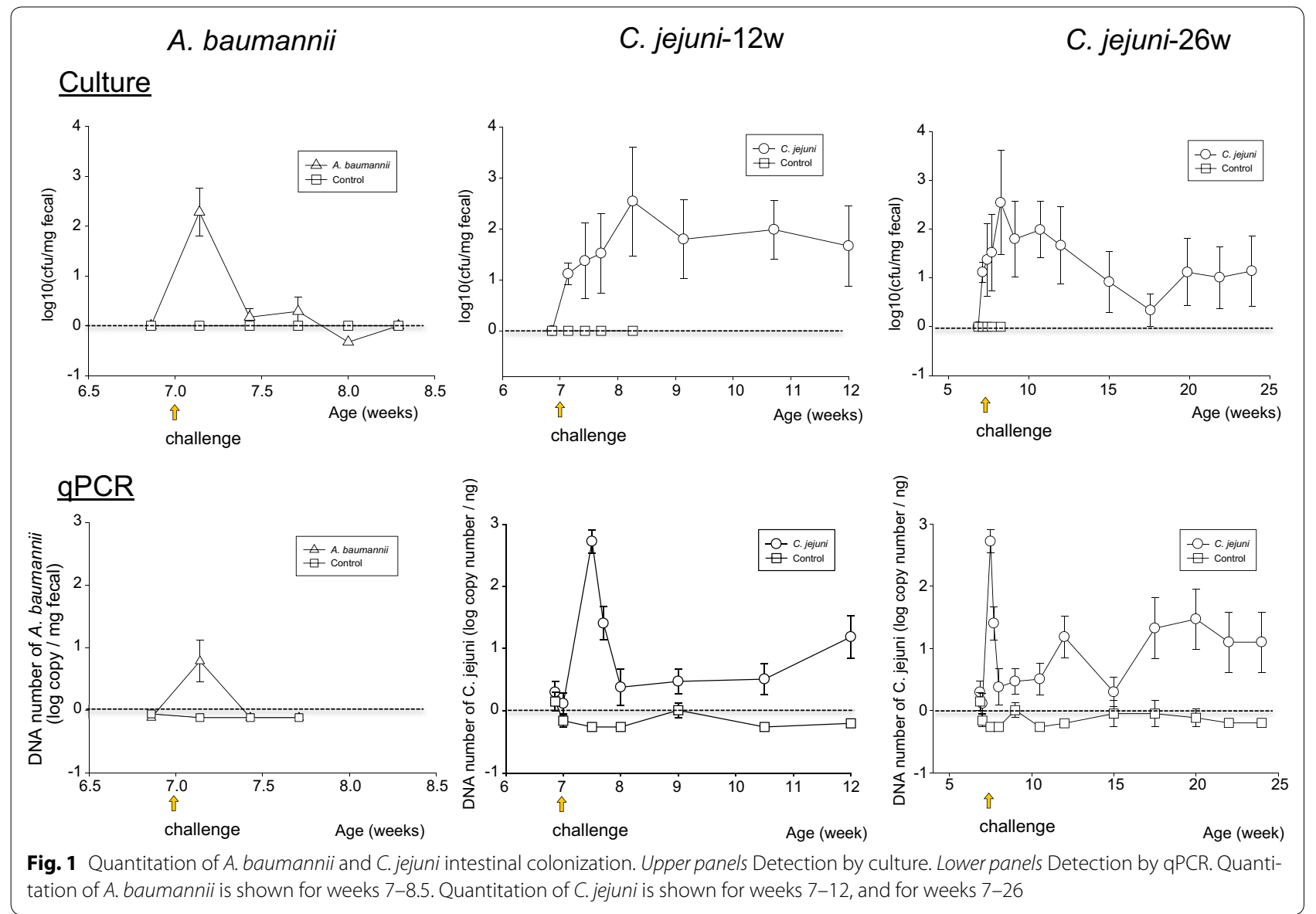

assessing the species richness of the gut microbiota, all three cages were similar, although at the final time point, the $C$. jejuni-challenged group showed lower richness than controls (column B). All three groups were similar in relative abundance at the genus level (column $\mathrm{C}$ ) except for late changes in S24-7 abundance in cage 2.

\section{Quantitative differences in specific taxa}

We next analyzed the HTS results using LEfSe, identifying specific taxa that showed significant differences between mice at the beginning (6.5 weeks of life) and end (23.9 weeks) of the experiment (Additional file 1: Figure S4). Multiple taxa within Firmicutes, Bacilli, and Tenericutes were significantly increased at the early time point in the three groups of mice with only cage-related minor variations. In contrast, taxa within Bacteroides and Verrucomicrobia were significantly higher at the later time point in all groups. Animals in cage 2 showed significantly increased Proteobacteria, consistent with our finding of persistent $C$. jejuni colonization. Proteobacteria were not increased in cage 1 (control) as expected, nor in cage 3 after $C$. jejuni colonization had spontaneously ceased.

\section{Detection of $C$. jejuni after mouse passage}

Next, we investigated whether three $C$. jejuni strains with different passage histories varied in their abilities to colonize the mouse gastrointestinal tract after oral challenge (Additional file 1: Figure S5). All three $C$. jejuni strains showed similar kinetics; culture (panel A) and qPCR

(See figure on next page.)

Fig. 2 Assessment of change in gut microbiome following C. jejuni challenge. Mice were challenged with either PBS (cage 1), or C. jejuni (cages 2 and 3) at 7 weeks of age. Fecal pellets were collected serially, DNA extracted, and HTS performed on the Illumina Miseq platform. $\beta$-diversity (column A) a-diversity (column B). and relative taxon abundance at the genus level (color-coded, column C) are shown. Light blue indicates control group (cage 1); dark blue indicates C. jejuni group (cage 2); red indicates another C. jejuni group (cage 3) 


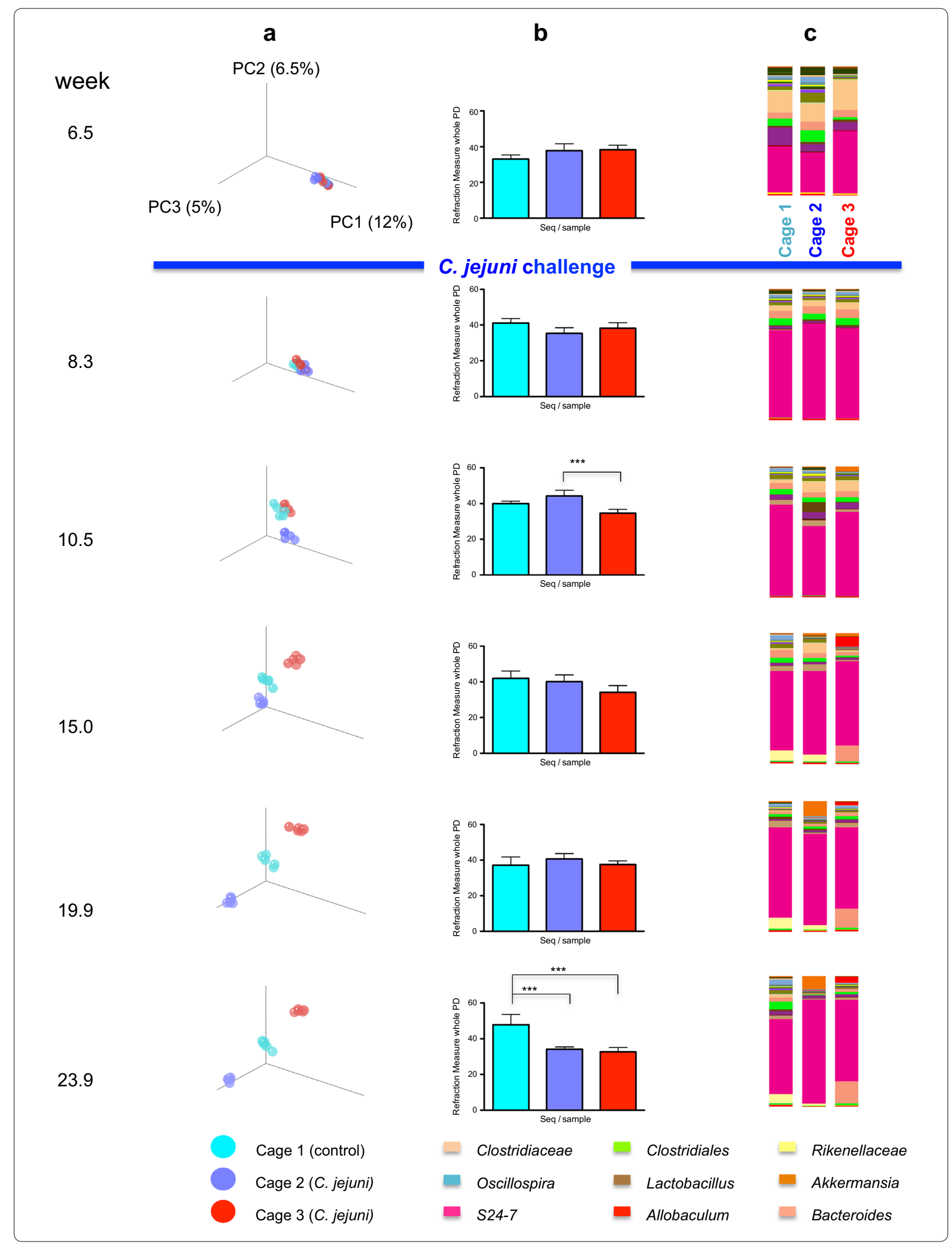


results (panel B) were consistent. Despite some variations in the kinetics of colonization, all three $C$. jejuni strains colonized the mouse gut to similar degrees.

\section{Effect of antibiotic treatment on mouse intestinal colonization}

We next studied the effect of pre-treatment of the mice with penicillin or ciprofloxacin, two antibiotics often used in clinical practice, on gut colonization with $C$. jejuni or A. baumannii. The antibiotics used to pre-treat the mice had no significant influence on intestinal colonization with either pathogen (Fig. 3). As in previous experiments, A. baumannii only transiently colonized the mice.

\section{Assessment of gut microbiota changes associated} with bacterial challenge and antibiotic pre-treatment

We assessed the gut microbiome in mice followed for nearly 9 weeks. Assessment of $\beta$-diversity of the gut microbiota in the group of mice with no antibiotic treatment had nearly identical community structure at 6 different time points, indicating stable populations. However, mice treated with penicillin showed dramatic changes in $\beta$-diversity, across time. In contrast, mice treated with ciprofloxacin had minor effects in $\beta$-diversity (Fig. 4, panel A).

We next performed analysis of $\beta$-diversity of the gut microbiota in relation to antibiotic treatment and pathogen challenge. As before, mice with no antibiotic treatment had nearly identical community structure, regardless of pathogen challenge. In the group of mice receiving ciprofloxacin, the no challenge group had minor changes in $\beta$-diversity compared to either the $A$. baumannii or $C$. jejuni challenged groups. As above, mice treated with penicillin showed dramatic changes in community structure. However, mice treated with penicillin and challenged with $C$. jejuni changed in $\beta$-diversity and then recovered immediately. Similar but less dramatic effects were observed in mice treated with penicillin and challenged with A. baumannii (Fig. 4, panel B).

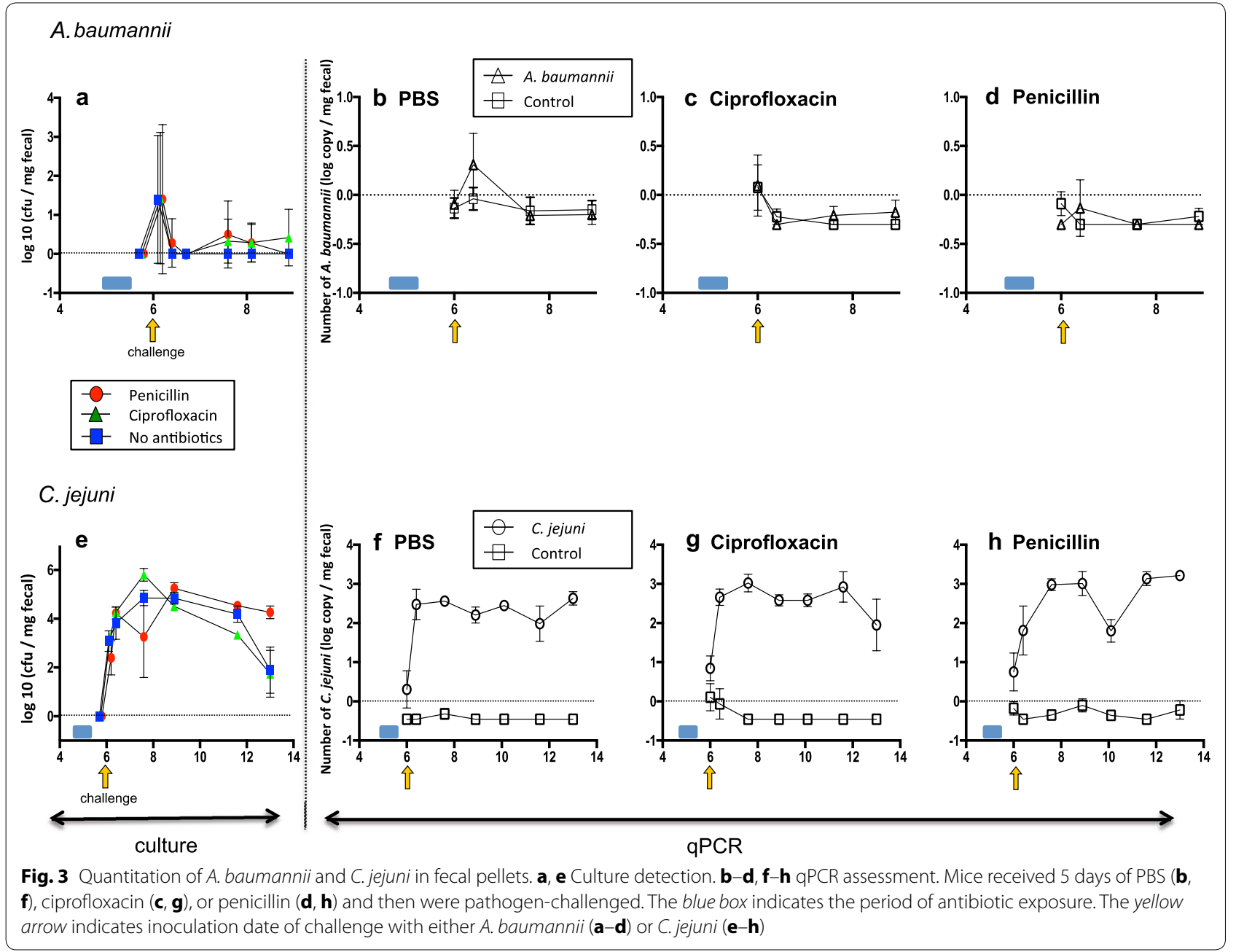



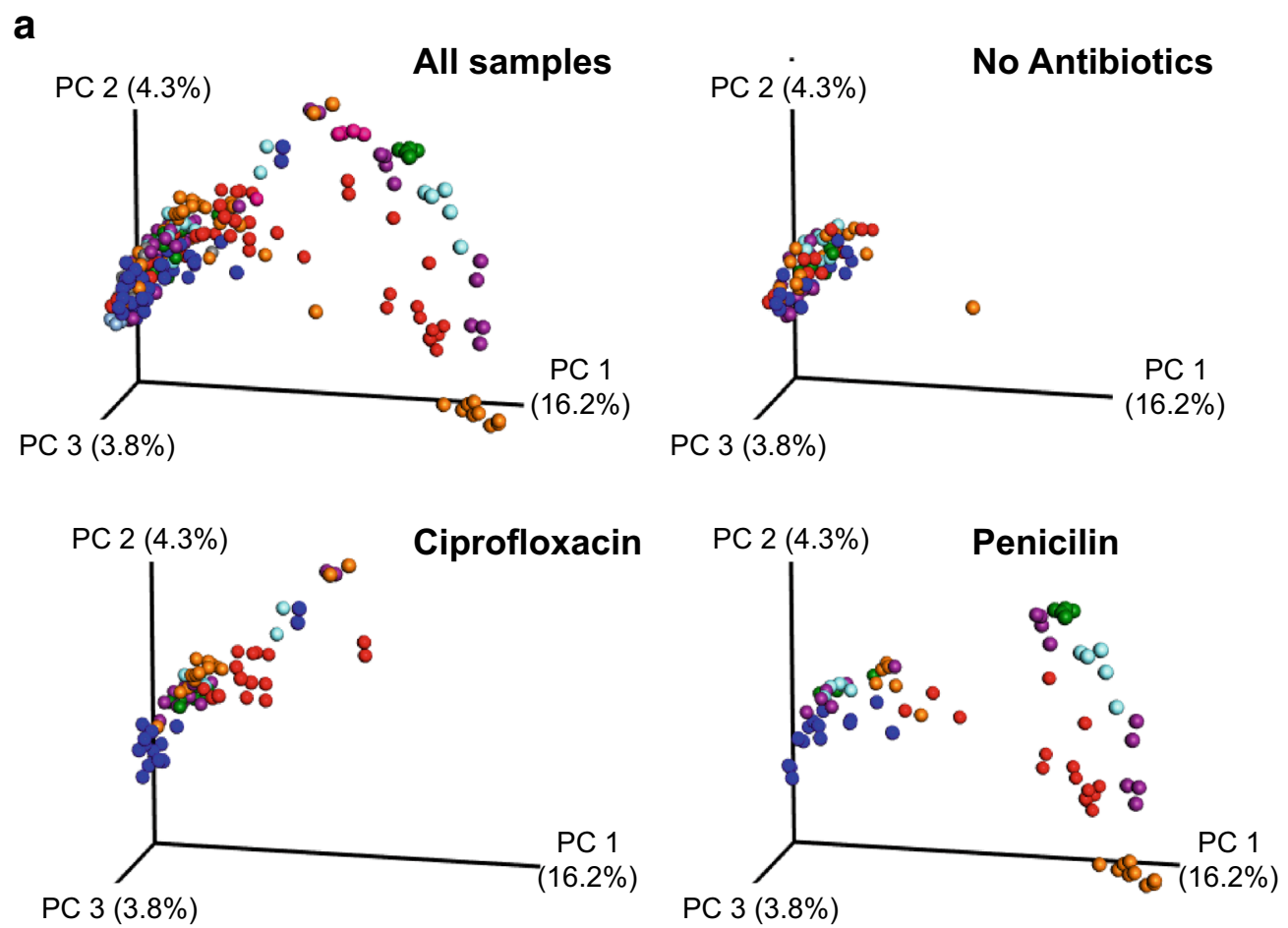

Figure 4. Panel A

b

Control
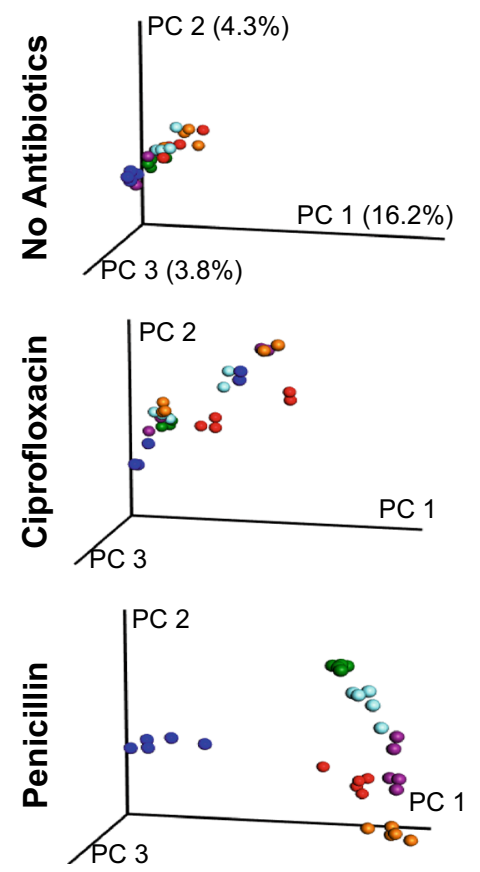

A. baumannii

C. jejuni
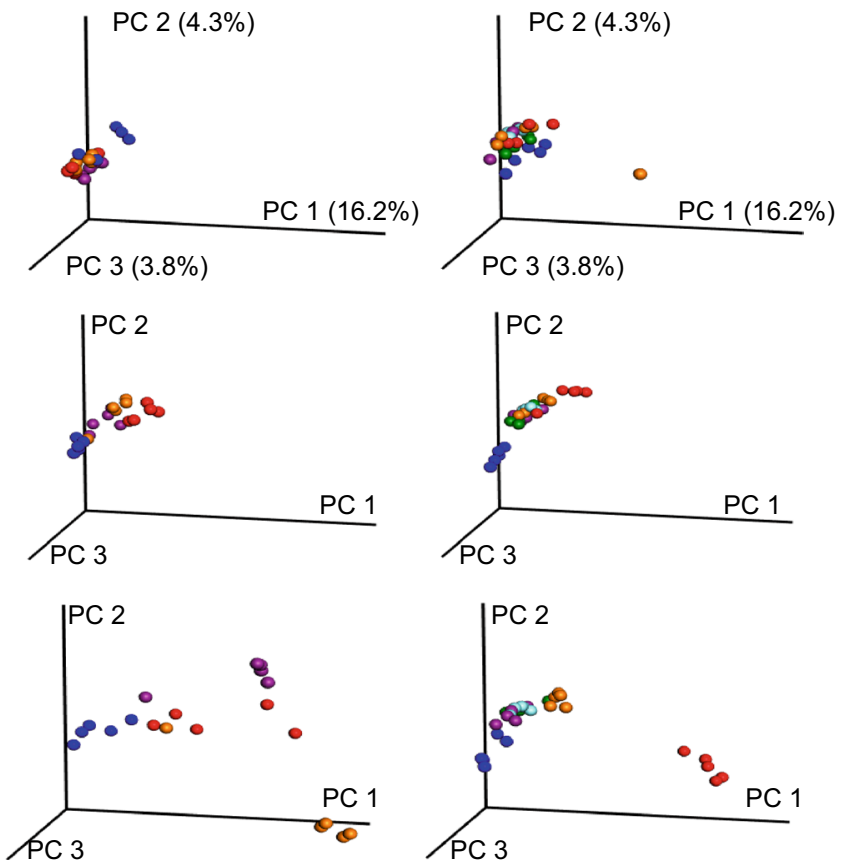
(See figure on previous page.)

Fig. 4 Assessment of antibiotic effects on intestinal microbial community structure ( $\beta$-diversity). PCoA of the Unweighted UniFrac distances of microbial 16S rRNA sequences (V4 region) in fecal samples at six time points is shown. Colored dots indicate composites of the microbial community at 4.3 weeks (blue; before antibiotic treatment), 5.4 weeks (red; current antibiotic treatment), 6.1 weeks (orange), 7.6 weeks (purple), 10.1 weeks (light blue), or 13.0 weeks (green), respectively. a PCoA analysis sorted by antibiotic treatment, $\mathbf{b}$ each column indicates the antibiotic treatment group, and each row represents the bacterial challenge

\section{Analysis of longitudinal inter-group phylogenic distances associated with bacterial challenge and antibiotic treatment}

We observed minor $\beta$-diversity differences of the gut microbiota in the different mouse groups at time 1 (4.3 week) which was before antibiotic treatment and pathogen challenge. As such, we considered that the difference may be related to cage effects at that time point (Additional file 1: Figure S6, panel A). Next, we analyzed the intergroup variability at different time points in relation to antibiotic treatment and pathogen challenge, using the PERMANOVA metric. We did not observe significant differences in community structure in the mice challenged with either $A$. baumannii or $C$. jejuni without antibiotic pre-treatment (control groups) at any time point. In contrast, community structure in the mice treated with antibiotics and challenged with $A$. baumannii were significantly different from community structure before challenge. Furthermore, community structure of antibiotic-treated mice that were challenged with $C$. jejuni also were significantly different than pre-challenge (Data not shown). The community structure differences in the mice treated with penicillin and challenged with $C$. jejuni were significantly greater than before challenge (Additional file 1: Figure S7). Interestingly, these data suggest that the presence of $C$. jejuni minimized microbiome susceptibility to penicillin effects.

In mice without antibiotic pre-treatment, $\alpha$-diversity was highest in the $C$. jejuni-challenged group; due to the short follow-up of the A. baumannii group, we could not assess changes. We consistently observed that in animals treated with penicillin, $\alpha$-diversity was significantly increased in those challenged with $C$. jejuni; ciprofloxacin did not affect richness (Additional file 1: Figure S6, panel B).

Finally, the relative abundance of taxa in the control mice (not pre-treated with antibiotics), that were challenged with A. baumannii or C. jejuni (Fig. 5, column A) differed little from the (control) mice without challenge. There was a major effect on taxa abundances in mice pretreated with penicillin, but this was significantly reduced after A. baumannii, or C. jejuni challenge (column C). In contrast, ciprofloxacin had much smaller effects (Fig. 5, column B).

\section{Discussion}

In these studies, persistent colonization was achieved with $C$. jejuni, an intestinal pathogen, but not with $A$. baumannii, consistent with prior reports [28, 29]. We assessed the health status of the mice challenged with the pathogens and with or without antibiotic treatment and we did not notice any significant change in behavior or signs of disturbances in the health of mice that confirmed previous information that mice do not express disease after colonization with $C$. jejuni [30, 31].

Longitudinal assessment of bacterial gut community structure indicates that at early time points, essentially all mice were identical at the phylum level. However, as the experiments progressed, community structures varied, due to cage effects, and independently in relation to antibiotic treatment, or $C$. jejuni challenge.

However, the community richness in the gut microbiome was relatively constant, unaffected by cage, or antibiotic effects. Nevertheless, C. jejuni challenge increased richness, which was affected by penicillin pre-treatment. Mouse-adapted strains of $C$. jejuni were not better than a parental strain in mouse gut colonization. One hypothesis for the mechanism for the increased richness is that C. jejuni competes with dominant strains, permitting the more minor taxa to bloom. An alternative hypothesis is that $C$. jejuni infection stimulates production of antimicrobial peptides, as has been reported [32], and these select against the dominant taxa.

Antibiotic pre-treatment showed no effect on the capabilities of $C$. jejuni and $A$. baumannii to colonize the mice, which for $A$. baumannii was only transient. We recognized that variation in antibiotic concentration may cause different effects. However, in this study we used antibiotics equivalent of therapeutic doses used in humans to reach maximum antibiotic concentration in mice. Stable community structure was observed in mice for nearly 10 weeks whether challenged or not. Ciprofloxacin induced minor community-wide disturbances but penicillin induced major disturbances (Fig. 5).

In the absence of antibiotic pre-treatment, pathogen introduction did not affect relative abundances of the colonizing taxa either at the phyla (Fig. 5) or genus level (Data not shown). In contrast, antibiotic pre-treatment with ciprofloxacin produced minor disturbance of the gut microbiota when compare to penicillin. One explanation 


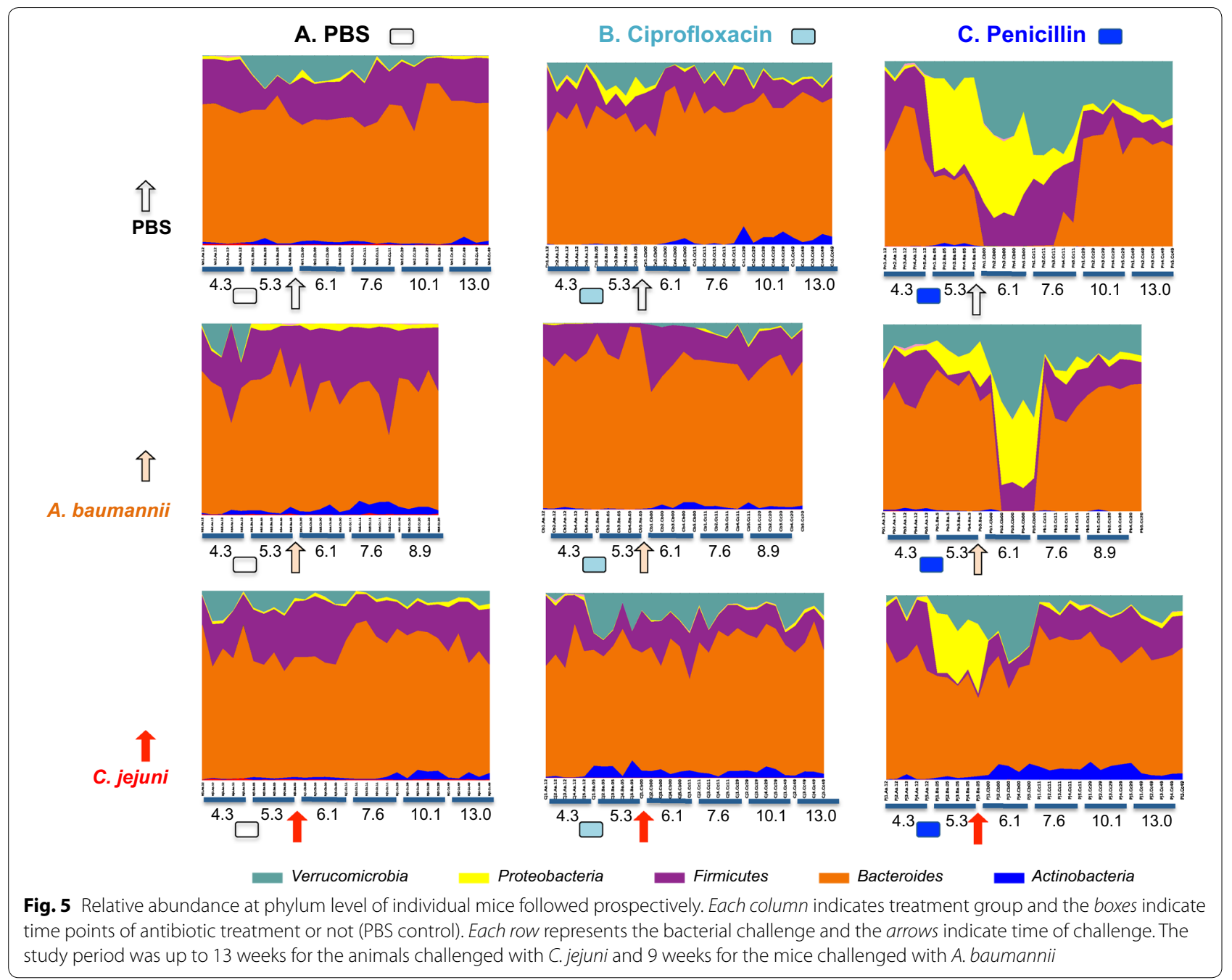

may be the lower activity of ciprofloxacin against anaerobes [33], in contrast to the known anti anaerobic activity of penicillin $[34,35]$. Surprisingly, the introduction of A. baumannii or C. jejuni ameliorated the disturbances in the community structure, and facilitated the recovery to normality. We speculate that pathogen introduction affected either host responses that led to stereotypic changes, or to altered competition dynamics in the gut, favoring the status quo ante.

In conclusion, the susceptibility to gut colonization by $A$. baumannii and $C$. jejuni did not change despite the disruption of the gut microbiota of mice treated with antibiotics. The distruption of the gut mictobiota may be reduced by C. jejuni colonization but the mechanism is still unknown.

\section{Additional file}

Additional file 1. Additional figures.

\section{Abbreviations}

HTS: high-throughput sequencing; rRNA: ribosomal ribonucleic acid; OTUs: operational taxonomic units; PCoA: principal coordinates analysis; LEfSE: linear discriminant analysis (LAD) effect size.

\section{Authors' contributions}

$\mathrm{TI}, \mathrm{TT}, \mathrm{WY}, \mathrm{ZG}$, and GV performed the experiments. TI and AVA conducted the bioinformatics analysis. GPP and MJB conceived the project. TI, GPP, and MJB wrote the manuscript. All authors read and approved the final manuscript.

\section{Author details}

${ }^{1}$ Department of Medicine, New York University School of Medicine, New York, NY, USA. ${ }^{2}$ Department of Agriculture, University of Miyazaki, Miyazaki, Japan. ${ }^{3}$ Universite Montpellier, Montpellier, France. ${ }^{4}$ Department of Microbiology, New York University School of Medicine, New York, NY, USA. ${ }^{5} 6026$ W VAMC, 423 East 23th Street, New York, NY 10010, USA.

\section{Acknowledgements}

Not applicable.

\section{Competing interests}

The authors declare that they have no competing interests.

\section{Availability of data and materials}

The data will be shared upon personal request. 


\section{Ethics approval and consent to participate}

IACUC protocol \#1 10504 was approved by New York University School of Medicine.

\section{Funding}

This work was supported in part by the US Army contract \#

W81XWH-11-1-0739, by the Diane Belfer Program in Human Microbial Ecology, the Ziff Family Fund, and the C \& D Fund. The funders had no role in this study.

Received: 14 October 2016 Accepted: 14 November 2016 Published online: 18 November 2016

\section{References}

1. Luckey TD. Introduction to intestinal microecology. Am J Clin Nutr. 1972:25(12):1292-4

2. Ley RE, Lozupone CA, Hamady M, Knight R, Gordon Jl. Worlds within worlds: evolution of the vertebrate gut microbiota. Nat Rev Microbiol. 2008;6(10):776-88.

3. Clemente JC, Ursell LK, Parfrey LW, Knight R. The impact of the gut microbiota on human health: an integrative view. Cell. 2012;148(6):1258-70.

4. Kamada N, Chen GY, Inohara N, Nunez G. Control of pathogens and pathobionts by the gut microbiota. Nat Immunol. 2013;14(7):685-90.

5. Buffie CG, Pamer EG. Microbiota-mediated colonization resistance against intestinal pathogens. Nat Rev Immunol. 2013;13(11):790-801.

6. Hooper LV, Macpherson AJ. Immune adaptations that maintain homeostasis with the intestinal microbiota. Nat Rev Immunol. 2010;10(3):159-69.

7. Hicks LA, Taylor TH Jr, Hunkler RJ. U.S. outpatient antibiotic prescribing, 2010. N Engl J Med. 2013;368(15):1461-2.

8. Bohnhoff M, Miller CP. Enhanced susceptibility to Salmonella infection in streptomycin-treated mice. J Infect Dis. 1962;111:117-27.

9. Robinson CJ, Young VB. Antibiotic administration alters the community structure of the gastrointestinal micobiota. Gut Microbes. 2010;1(4):279-84

10. Buffie CG, Bucci V, Stein RR, McKenney PT, Ling L, Gobourne A, No D, Liu H, Kinnebrew M, Viale A, et al. Precision microbiome reconstitution restores bile acid mediated resistance to Clostridium difficile. Nature. 2015:517(7533):205-8

11. Cho I, Yamanishi S, Cox L, Methe BA, Zavadil J, Li K, Gao Z, Mahana D, Raju $\mathrm{K}$, Teitler I, et al. Antibiotics in early life alter the murine colonic microbiome and adiposity. Nature. 2012;488(7413):621-6.

12. Cox LM, Yamanishi S, Sohn J, Alekseyenko AV, Leung JM, Cho I, Kim SG, $\mathrm{Li} \mathrm{H}, \mathrm{Gao}$ Z, Mahana D, et al. Altering the intestinal microbiota during a critical developmental window has lasting metabolic consequences. Cell. 2014;158(4):705-21

13. Wagenaar JA, French NP, Havelaar AH. Preventing Campylobacter at the source: why is it so difficult? Clin Infect Dis. 2013;57(11):1600-6.

14. Dasti Jl, Tareen AM, Lugert R, Zautner AE, Gross U. Campylobacter jejuni: a brief overview on pathogenicity-associated factors and disease-mediating mechanisms. Int J Med Microbiol. 2010;300(4):205-11.

15. Allos BM. Campylobacter jejuni infections: update on emerging issues and trends. Clin Infect Dis. 2001;32(8):1201-6.

16. Gulen TA, Guner R, Celikbilek N, Keske S, Tasyaran M. Clinical importance and cost of bacteremia caused by nosocomial multi drug resistant Acinetobacter baumannii. Int J Infect Dis. 2015;38:32-5.
17. Martin-Loeches I, Diaz E, Valles J. Risks for multidrug-resistant pathogens in the ICU. Curr Opin Crit Care. 2014;20(5):516-24.

18. Ayats J, Corbella X, Ardanuy C, Dominguez MA, Ricart A, Ariza J, Martin $\mathrm{R}$, Linares J. Epidemiological significance of cutaneous, pharyngeal, and digestive tract colonization by multiresistant Acinetobacter baumannii in ICU patients. J Hosp Infect. 1997;37(4):287-95.

19. Korlath JA, Osterholm MT, Judy LA, Forfang JC, Robinson RA. A pointsource outbreak of campylobacteriosis associated with consumption of raw milk. J Infect Dis. 1985:152(3):592-6.

20. Black RE, Levine MM, Clements ML, Hughes TP, Blaser MJ. Experimental Campylobacter jejuni infection in humans. J Infect Dis. 1988;157(3):472-9.

21. Gao Z, Perez-Perez Gl, Chen Y, Blaser MJ. Quantitation of major human cutaneous bacterial and fungal populations. J Clin Microbiol. 2010;48(10):3575-81.

22. He Y, Chen CY. Quantitative analysis of viable, stressed and dead cells of Campylobacter jejuni strain 81-176. Food Microbiol. 2010;27(4):439-46.

23. Chuang YC, Chang SC, Wang WK. High and increasing Oxa-51 DNA load predict mortality in Acinetobacter baumannii bacteremia: implication for pathogenesis and evaluation of therapy. PLoS ONE. 2010;5(11):e14133.

24. Caporaso JG, Kuczynski J, Stombaugh J, Bittinger K, Bushman FD, Costello EK, Fierer N, Pena AG, Goodrich JK, Gordon Jl, et al. QIIME allows analysis of high-throughput community sequencing data. Nat Methods. 2010;7(5):335-6.

25. Edgar RC. Search and clustering orders of magnitude faster than BLAST. Bioinformatics. 2010;26(19):2460-1.

26. Caporaso JG, Lauber CL, Walters WA, Berg-Lyons D, Lozupone CA, Turnbaugh PJ, Fierer N, Knight R. Global patterns of 16S rRNA diversity at a depth of millions of sequences per sample. Proc Natl Acad Sci USA. 2011:108(Suppl 1):4516-22.

27. Segata N, Izard J, Waldron L, Gevers D, Miropolsky L, Garrett WS, Huttenhower C. Metagenomic biomarker discovery and explanation. Genome Biol. 2011;12(6):R60

28. Seifert $H$, Baginski $R$, Schulze A, Pulverer $G$. The distribution of Acinetobacter species in clinical culture materials. Zentralbl Bakteriol. 1993;279(4):544-52

29. Luna CM, Aruj PK. Nosocomial Acinetobacter pneumonia. Respirology. 2007;12(6):787-91.

30. Blaser MJ, Duncan DJ, Warren GH, Wang WL. Experimental Campylobacter jejuni infection of adult mice. Infect Immun. 1983;39(2):908-16.

31. Stanfield JT, McCardell BA, Madden JM. Campylobacter diarrhea in an adult mouse model. Microb Pathog. 1987;3(3):155-65.

32. Lohans CT, van Belkum MJ, Li J, Vederas JC. Characterization of bacterial antimicrobial peptides active against Campylobacter jejuni. Can J Chem. 2015:93(4):381-8

33. Behra-Miellet J, Dubreuil L, Jumas-Bilak E. Antianaerobic activity of moxifloxacin compared with that of ofloxacin, ciprofloxacin, clindamycin, metronidazole and beta-lactams. Int J Antimicrob Agents. 2002;20(5):366-74.

34. Tally FP, Jacobus NV, Bartlett JG, Gorbach SL. In vitro activity of penicillins against anaerobes. Antimicrob Agents Chemother. 1975;7(4):413-4.

35. Bryskier A. Anti-anaerobic activity of antibacterial agents. Expert Opin Investig Drugs. 2001;10(2):239-67.

\section{Submit your next manuscript to BioMed Central and we will help you at every step:}

- We accept pre-submission inquiries

- Our selector tool helps you to find the most relevant journal

- We provide round the clock customer support

- Convenient online submission

- Thorough peer review

- Inclusion in PubMed and all major indexing services

- Maximum visibility for your research

Submit your manuscript at www.biomedcentral com/submit
Ciomed Central 